\title{
Stochastic modelling for biodosimetry: Predicting the chromosomal response to radiation at different time points after exposure ${ }^{\star}$
}

\author{
Joanna Deperas-Standylo ${ }^{1}$, Ewa Gudowska-Nowak ${ }^{1, a}$, and Sylvia Ritter ${ }^{2}$ \\ 1 Mark Kac Complex Systems Research Center and Marian Smoluchowski Institute of Physics, Jagiellonian University \\ in Kraków, Poland \\ 2 GSI Helmholtzzentrum für Schwerionenforschung, Planckstrasse 1, 64291 Darmstadt, Germany
}

Received 6 January 2014 / Received in final form 15 April 2014

Published online 29 July 2014

(C) The Author(s) 2014. This article is published with open access at Springerlink.com

\begin{abstract}
Cytogenetic data accumulated from the experiments with peripheral blood lymphocytes exposed to densely ionizing radiation clearly demonstrate that for particles with linear energy transfer (LET) $>100 \mathrm{keV} / \mu \mathrm{m}$ the derived relative biological effectiveness (RBE) will strongly depend on the time point chosen for the analysis. A reasonable prediction of radiation-induced chromosome damage and its distribution among cells can be achieved by exploiting Monte Carlo methodology along with the information about the radius of the penetrating ion-track and the LET of the ion beam. In order to examine the relationship between the track structure and the distribution of aberrations induced in human lymphocytes and to clarify the correlation between delays in the cell cycle progression and the aberration burden visible at the first post-irradiation mitosis, we have analyzed chromosome aberrations in lymphocytes exposed to Fe-ions with LET values of $335 \mathrm{keV} / \mu \mathrm{m}$ and formulated a Monte Carlo model which reflects time-delay in mitosis of aberrant cells. Within the model the frequency distributions of aberrations among cells follow the pattern of local energy distribution and are well approximated by a time-dependent compound Poisson statistics. The cell-division cycle of undamaged and aberrant cells and chromosome aberrations are modelled as a renewal process represented by a random sum of (independent and identically distributed) random elements $S_{N}=\sum_{i=0}^{N} X_{i}$. Here $N$ stands for the number of particle traversals of cell nucleus, each leading to a statistically independent formation of $X_{i}$ aberrations. The parameter $N$ is itself a random variable and reflects the cell cycle delay of heavily damaged cells. The probability distribution of $S_{N}$ follows a general law for which the moment generating function satisfies the relation $\Phi_{S_{N}}=\Phi_{N}\left(\Phi_{X_{i}}\right)$. Formulation of the Monte Carlo model which allows to predict expected fluxes of aberrant and non-aberrant cells has been based on several input information: (i) experimentally measured mitotic index in the population of irradiated cells; (ii) scored fraction of cells in first cell cycle; (iii) estimated average number of particle traversals per cell nucleus. By reconstructing the local dose distribution in the biological target, the relevant amount of lesions induced by ions is estimated from the biological effect induced by photons at the same dose level. Moreover, the total amount of aberrations induced within the entire population has been determined. For each subgroup of intact (non-hit) and aberrant cells the cell-division cycle has been analyzed reproducing correctly an expected correlation between mitotic delay and the number of aberrations carried by a cell. This observation is of particular importance for the proper estimation of the biological efficiency of ions and for the estimation of health risks associated with radiation exposure.
\end{abstract}

\section{Introduction}

Biological effects of ionizing radiation result primarily from damage to DNA which contains genetic information crucial for functioning and division of the cell. Ionizing

\footnotetext{
* Contribution to the Topical Issue "Nano-scale Insights into Ion-beam Cancer Therapy", edited by Andrey V. Solov'yov, Nigel Mason, Paulo Limão-Vieira and Malgorzata SmialekTelega.

a e-mail: gudowska@th.if.uj.edu.pl
}

radiation induces several types of DNA damages such as single- and double-strand breaks (SSBs and DSBs, respectively), base damages and DNA-protein cross links (cf. Fig. 1). Among these, DSBs are considered to be the most severe radiation-induced damage determining the fate of the injured cell, since non-repaired or misrepaired DSBs can give rise to chromosome aberrations, mutations or cell transformation and cell death. Experimental data and modelling studies, both evidence that the quality of lesions changes as a function of LET. Whereas for low-LET radiation a random energy deposition results in a uniformly 


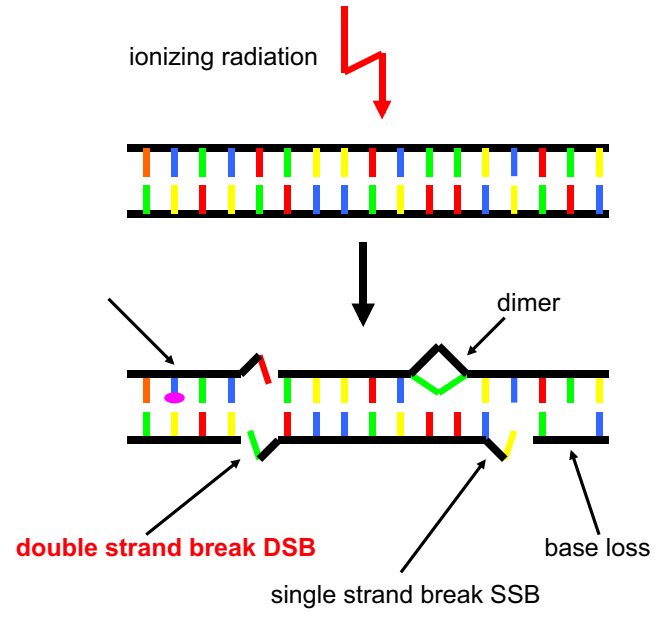

Fig. 1. DSB is a key cytogenetic lesion for ionizing radiation. It is generated when two complementary strands of DNA double helix become broken simultaneously at sites which are distant apart by several base pairs. Proximity of breakage is responsible for disruption of the DNA structure.

distributed pattern of lesions, for high-LET particle radiation the localized dose deposition along the track of a projectile leads to a non-random clustering of radiation events and subsequent damage induction which can be correlated over short distances. Intricate clusters of damage formed in this way comprise one or more DSBs, as well as associated SSBs, base damages and cross links within about ten base pairs. Because of their complexity, such clusters are less repairable than sparsely distributed single damages.

Since the discovery of radiation and radioactivity more than 100 years ago, its beneficiary application for the mankind ranges from medicine - where both conventional low LET radiation (photons) and high LET ion beams are used for cancer therapy - to industry, agriculture and research. On the other hand, understanding biological effects of irradiation is a crucial factor in radioprotection programmes aimed to lower potential risks associated with the use of radiation sources, occupational hazard and accidental exposures [1-3]. Radioprotection management is based on evaluation of dosimetric quantities, like dose, by use of dedicated physical dosimeters. However, in case of accidental exposures of non-professionals, dosimetric evaluations of absorbed doses have to rely mainly on biomarker studies in which the assessment of dose is related to specific biological endpoints of interest. In a standard biodosimetric method information about previous exposure to radiation is gained by detecting and analyzing chromosome aberrations whose score correlates with the absorbed dose. Notably, dose-effect relationship has a different character for low- and high-LET radiation, manifested in shapes and slopes of derived induction curves.

On Earth, the natural and artificial radiation results mainly from sparsely (low-LET) ionizing radiations such as $\mathrm{X}_{-}^{-}, \beta$ or $\gamma$-rays, while in deep space high energy protons, He-ions and heavier ions named high-Z and high energy particles predominate. Although particles like Fe-ions contribute only with 1 percent to the overall particle flux in space, they are a major concern for astronaut safety, due to their high ionizing energy and great penetration power [4]. Up to date, only limited data on the biological effects of heavy, high energetic particles are available leading to large uncertainties in the prediction of adverse health effects such as the induction of cancer, neurological disorders or cataracts $[5,6]$.

To contribute to this issue we investigate the induction of chromosomal damage in peripheral human blood lymphocytes after exposure to Fe- particles by means of stochastic modelling and Monte Carlo simulations based on experimental measurements. The main purpose of the modelling described in the forthcoming sections is to establish the procedure which would allow us to (i) predict aberration yield per one particle traversal; and (ii) estimate expected distribution of damage among cycling cells at different times after exposure.

\section{Materials and methods}

Lymphocytes were isolated from the whole blood, resuspended at a density of $4 \times 10^{6}$ cells $/ \mathrm{mL}$ in complete medium (RPMI 1640 medium supplemented with $20 \%$ fetal calf serum, $2 \mathrm{mM}$ L-glutamine, $100 \mathrm{U} / \mathrm{mL}$ penicillin, $100 \mu \mathrm{g} / \mathrm{mL}$ streptomycin) as described in detail elsewhere [7].

All exposures were done at GSI. Irradiation with Feions (initial energy: $200 \mathrm{MeV} / \mathrm{u}$ ) was performed at the heavy ion synchrotron SIS in specially designed polyethylene holders with a 2-mm-thick well for the sample and 1-mm plastic between the cells and the radiation source. At sample position the energy of the Fe-ions was $177 \mathrm{MeV} / \mathrm{u}$ with LET $=335 \mathrm{keV} / \mu \mathrm{m}$. By energy loss calculations it was confirmed that track segment conditions are fulfilled. For comparison, cells were irradiated with X-rays $(250 \mathrm{kV}, 16 \mathrm{~mA}, 1 \mathrm{~mm} \mathrm{Al}$ and $1 \mathrm{~mm} \mathrm{Cu}$ filtering).

Particle and X-ray exposures were done at room temperature and controls were sham irradiated. For Fe ions (2.2 Gy), the irradiation time was about $2 \mathrm{~min}$. The exposures to X-rays were performed at a dose rate of $2 \mathrm{~Gy} / \mathrm{min}$.

Preparation of metaphase cells, chromosome staining and aberration analysis have been performed according to standard techniques. Chromosome spreads have been prepared and stained with Fluorescence-plus-Giemsa (FPG) technique to distinguish between metaphases in first, second and third post-irradiation cycles [8,9]. All aberration types detectable with the staining method have been scored, including chromatid-type aberrations (for further discussion, see $[10,11])$. Througout the paper we use experimental data sets published in reference [9].

\section{Statistical analysis: number of aberrations induced by multiple particle traversals}

We refer to a random variable $X_{i}$ which represents number of aberrations induced by $i$ th single particle traversal through the target (a cell nucleus). After assuming that all particle traversals are statistically independent events, 


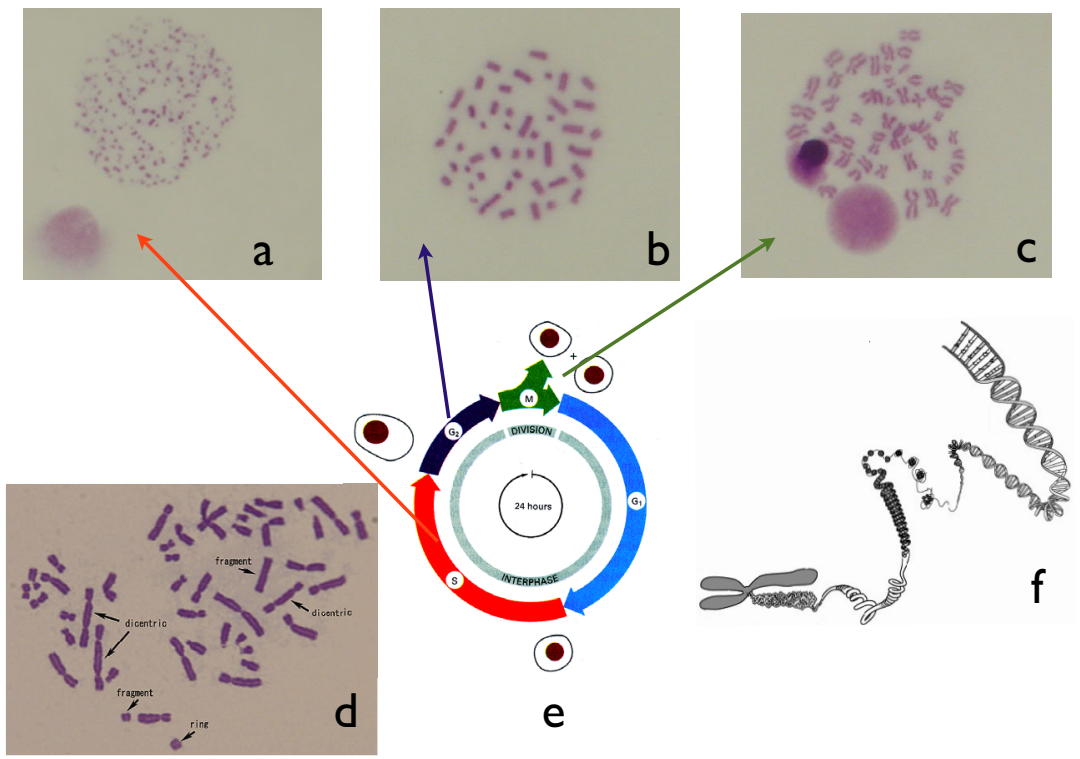

Fig. 2. Chart explaining detection and analysis of radiation-induced chromosome aberrations in a standard Giemsa staining: artificial induction of cell proliferation is followed by subsequent processing of DNA lesions in course of DNA synthesis in the S- and G-phases (a), (b) of the cell-cycle (e). Arrest of the cells in mitosis (c) allows to analyzed structural chromosome abnormalities (d). Formation of chromosomes involves complex chromatin folding and packing (f) in an interphase nucleus of typically $5-20 \mu \mathrm{m}$ in diameter. we analyze the random sum of identically distributed and independent (i.i.d.) stochastic elements $S_{N}=\sum_{i=0}^{N} X_{i}$ which, by definition, is itself a random variable [12]. In this formalism $N$ stands for a (random, and in general, time dependent) number of particle hits per nucleus whose distribution can be described by the probability generating function (p.g.f.) $F_{N}(s) \equiv E\left(s^{N}\right)=\sum_{n=0}^{\infty} P(N=$ $n) s^{n}$, where $F: \mathbb{R} \rightarrow \mathbb{R}$ and $s$ stands for the argument of the function $F$. Since $X_{i}$ is i.i.d., the p.g.f. is $F_{X_{i}}(s)=E\left(s^{X_{i}}\right)=\sum_{k=0}^{\infty} P\left(X_{i}=k\right) s^{k}$. By using the Bayes rule and statistical independence of $X_{i}$, the corresponding p.g.f. of $S_{N}$ variable can be recast in the form of:

$$
F_{S_{N}}(s)=E\left(s^{S_{N}}\right)=\sum_{n=0}^{\infty} F_{X_{i}}^{n}(s) P(N=n)=F_{N}\left(F_{X_{i}}(s)\right) .
$$

Accordingly, by differentiation of the $F_{S_{N}}(s)$, the information about p.m.f. can be retrieved from the formula

$$
P\left(S_{N}=k\right)=\left.\frac{F_{S_{N}}^{(k)}(s)}{k !}\right|_{s=0} .
$$

With the known $P(N=n)$ and for $k=0$, the above equation takes the form:

$$
\begin{aligned}
P\left(S_{N}=0\right) & =\sum_{n=0}^{\infty} P(N=n)\left(\sum_{j=0}^{\infty} P\left(X_{i}=j\right) 0^{j}\right)^{n} \\
& =\sum_{n=0}^{\infty} P(N=n)\left(P\left(X_{i}=0\right)\right)^{n} .
\end{aligned}
$$

Obviously, for known distributions $P\left(S_{N}=k\right), P(N=n)$ (referring to the overall number of damage produced due to $N$ particle traversals through the nucleus), recurrent use of equation (3) allows to derive distribution $P\left(X_{i}=x\right)$ for $x=0,1,2 \ldots$ For example, be deriving $P\left(X_{i}=0\right)$ and
$P\left(X_{i}=1\right)$ from equation (3), in the next step one can evaluate

$P\left(S_{N}=1\right)=P\left(X_{i}=1\right) \sum_{n=1}^{\infty} n P(N=n)\left(P\left(X_{i}=0\right)\right)^{n-1}$.

Assuming that $E(N)=\lambda$ is an average number of particle traversals per nucleus and $E\left(X_{i}\right)=\mu$ - an average number of aberrations induced by one particle traversal with corresponding variances $\operatorname{Var}(N)=\tau^{2}$ and $\operatorname{Var}\left(X_{i}\right)=\sigma^{2}$, the overall mean and variance of aberrations produced in one cell nucleus can be easily derived from equation (1):

$$
\begin{aligned}
E\left(S_{N}\right) & =\frac{d F_{S_{N}}}{d s}(1)=\mu \lambda \\
\operatorname{Var}\left(S_{N}\right) & =\frac{d^{2} F_{S_{N}}}{d s}(1)+\frac{d F_{S_{N}}}{d s}(1)-\left(\frac{d F_{S_{N}}}{d s}(1)\right)^{2} \\
& =\lambda \sigma^{2}+\mu^{2} \tau^{2}
\end{aligned}
$$

In practice, random distribution of particle traversals through the nucleus is well approximated by the Poisson statistics $[13-16]$ with the characteristic mean value of particle hits $E(N)=\lambda$ related to the dose imparted to the target. As a consequence, the formula equation (1) can be rewritten in the form representative for the Neyman-type A statistics [11,17-19]

$$
F_{S_{N}}(s)=e^{-\lambda\left(1-F_{X_{i}}(s)\right)} .
$$

\section{Aberration yield in normal human lymphocytes}

\subsection{Track structure}

The observed radiation damage results from a local spatial distribution of ionization events responsible for inducing DSBs and followed by formation of aberrations (see Figs. 1 and 2). For X-rays the energy deposition in the 

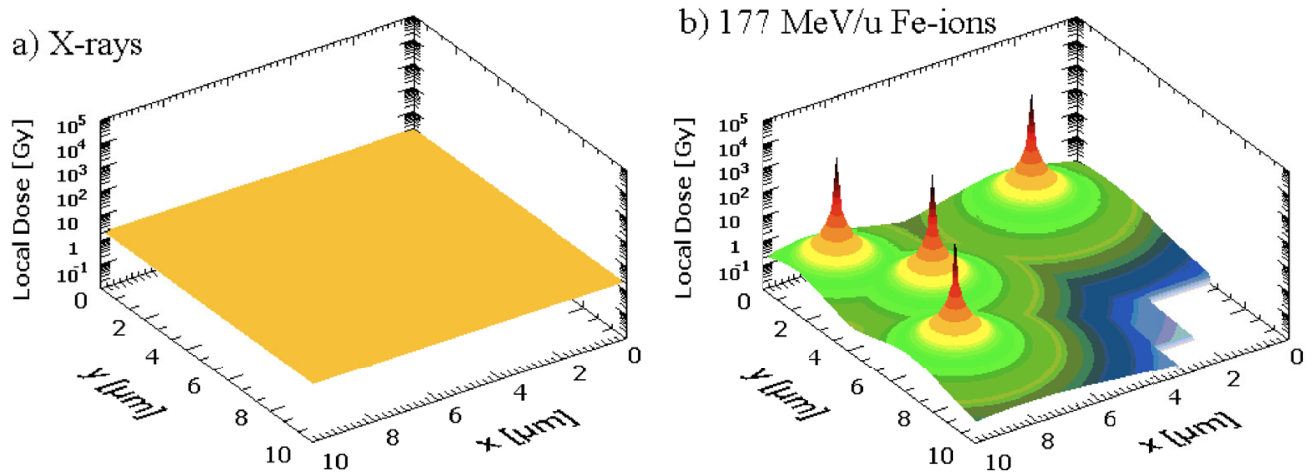

Fig. 3. Local dose deposition of photons and heavy ions examined in the present study: (a) 2 Gy of $\mathrm{X}-\mathrm{rays}(\mathrm{LET}=2 \mathrm{keV} / \mu \mathrm{m})$ and (b) $2.2 \mathrm{~Gy}$ of $177 \mathrm{MeV} / \mathrm{u}$ Fe-ions $\left(3.7 \times 10^{6} \mathrm{ions} / \mathrm{cm}^{2}, \mathrm{LET}=335 \mathrm{keV} / \mu \mathrm{m}\right)$. The radial dose profiles of tracks were calculated by a software developed at GSI. For Fe-ions the track radius scales with the energy of a projectile like $R \propto E^{1.7}$ and is $\approx 330 \mu \mathrm{m}$. A fluence of $4 \times 10^{6}$ ions $/ \mathrm{cm}^{2}$ corresponds to about 1 direct hit per cell nucleus of a human lymphocyte.

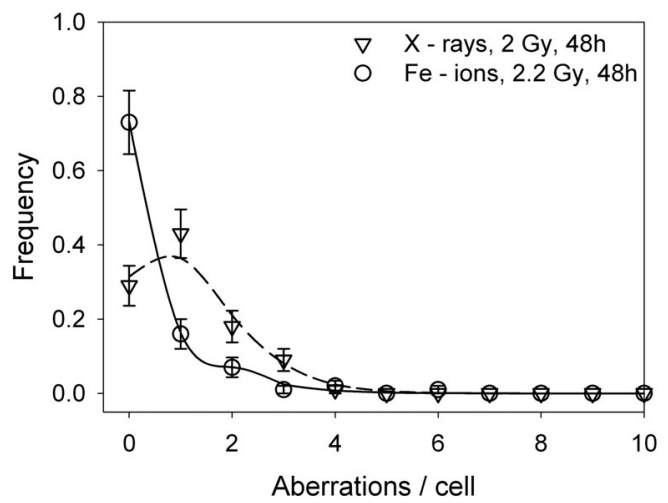

Fig. 4. Distribution of chromosome aberrations induced by 2 Gy of X-rays and 2.2 Gy of $177 \mathrm{MeV} / \mathrm{u}$ Fe-ions in 100 cells scored at $48 \mathrm{~h}$ after exposure to radiation. The relative frequency of first cycle metaphases carrying a distinct number of aberrations is plotted. Error bars on the data represent sample variations and have been estimated as $\sqrt{n}$, where $n$ is the number of events of a given class. The solid line represents the best fit to the experimental data by either the Poisson (X-rays) or Neyman-type A (ions) distributions.

micrometer scale (adequate to the size of the cell nucleus) is homogeneous, cf. Figure 3a. In line with this observation, the frequency distribution of damages can be then well fitted by use of the Poisson statistics $P(x)=\frac{\langle x\rangle^{x} e^{-\langle x\rangle}}{x !}$ with the mean value $\langle x\rangle$ (cf. Fig. 4).

In contrast, irradiation with a similar dose of Fe-ions results in a microscopic energy distribution with a clear non-uniform pattern, cf. Figure 3b. Charged particles deposit the energy along their trajectory with a distinct fall off of the dose at a distance $r$ from their path showing a $1 / r^{2}$ dependence $[14,16,20]$. The maximum radial range of $\delta$ electrons is limited and defines the corresponding track radius $R$ with a power-law dependence on the specific energy $E$ of the projectile $R \approx 0.05 \times E^{1.7}$, where $R$ is measured in $\mu \mathrm{m}$ and $E$ in $\mathrm{MeV} / \mathrm{u}$. The pattern of induced ionizations is reflected in the distribution of primary lesions (DSBs) in cell nuclei crossed by an ion and can

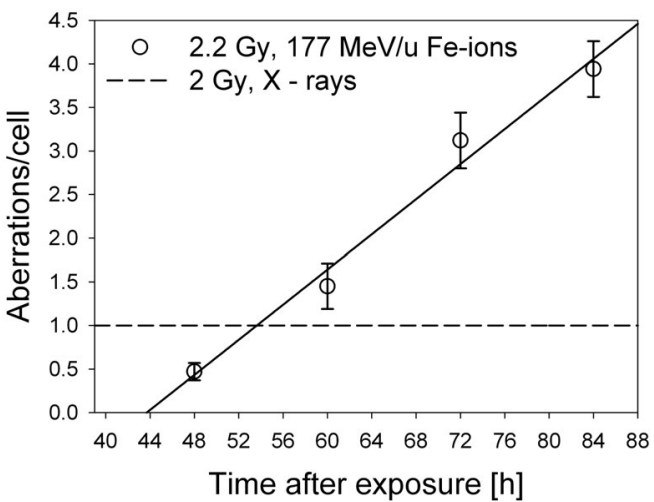

Fig. 5. Amount of aberrations per cell observed in first metaphases at different times after exposure to 2.2 Gy of $177 \mathrm{MeV} / \mathrm{u}$ Fe-ions $(335 \mathrm{keV} / \mu \mathrm{m})$. Error bars are SEM (standard error of the mean). For comparison, the dashed line represents a constant, time-independent aberration yield observed after exposure to 2 Gy X-rays $[9,11]$.

be visualized by immunochemical techniques [21]. Altogether, due to the stochastics of particle hits ion exposure results in a considerably inhomogeneous and dispersed distribution of aberrations among cells since non-hit cells and cells with different number of traversals are present in the same population, cf. Figure 4.

For human lymphocytes the mean geometrical crosssection of the cell nucleus is about $25 \mu \mathrm{m}^{2}$ [22] and the nuclear region forms approximately $65 \%$ of the total cell area. Consequently, for lymphocytes an exposure to a particle fluence $4 \times 10^{6}$ ions $/ \mathrm{cm}^{2}$ leads to a mean number of one direct particle traversal per cell nucleus. Therefore, knowing the fluence of the ion beam the average number of particle traversals per cell nucleus, $\lambda$ can be estimated.

\subsection{Impact of aberration burden on progression of cells in mitosis}

In Figure 5 the chromosomal damage measured in lymphocytes reaching the first mitosis by $48,60,72$ and $84 \mathrm{~h}$ 
has been plotted. In lymphocytes irradiated with X-rays with doses up to 2 Gy the aberration frequency in first cycle metaphases is almost constant in time $[9,10,23]$. In contrast, after exposure to particles with higher LET values, the aberration frequency elevates markedly with time, i.e. cells passaged by many particles are found to reach mitosis later than those with a lower number of hits or unhit cells $[11,24-27]$. The significant differences in aberration yields observed at earlier and later culture times (cf. Fig. 5) can therefore complicate the estimation of absorbed doses as e.g. used in biological dosimetry.

Cell population grows as cells progress to mitosis and divide. Therefore, in order to analyze how irradiated cells differ in their capabililty to divide, fluxes of aberrant and non-aberrant cells should be examined in the growing population with respect to an initial number of cells [10,27-30]. By using multiple-sampling technique [31] and a mathematical folding of the data obtained at different time-intervals the integrated fluxes of aberrant cells and aberrations detected in mitosis can be analyzed [9-11] and the loss of heavily damaged cells from irradiated cell populations can be properly estimated.

In forthcoming sections we introduce modifications of a previously proposed kinetic reaction-rate models [28,32-35] which incorporated cell-cycle delay effects in the prediction of aberration yield observed in samples of growing population of cells at various time intervals after exposure. In those approaches, owing to the observation that each sample reflects only the damage produced within a segment of the cell population, the total amount of aberrant cells and aberrations are determined by integration of fluxes of aberrant cells (or aberrations) visible in mitosis at a given time. In other words, for each collection time, the yield of aberrant cells and aberrations is weighted with the corresponding mitotic index reflecting kinetic rate of the cells (the tempo of passage through mitosis). Because of experimental limitations (passage of cells is recorded not continuously but only at several time points), a linear interpolation between data points has to be performed. Moreover, in order to consider the dilution of observed aberration yields in increased number of cells proliferating during the experiment, a correction factor for the mitotic index has to be applied.

Based on such analysis, accumulated data are summed yielding the realistic estimates of fraction of aberrant cells, the number of aberrations induced per 100 initial cells and the proportion of lost cells (i.e. cells which do not reach the first mitosis) [28,32-34]. Further modification of the model allows to predict the distribution of aberrations among cells at various times after exposure.

\section{Time-dependent aberration yield as a stochastic process}

\subsection{MI model (mitotic index model)}

Let $q(t)$ be the probability that cell hit by one particle has survived and is able to reach mitosis at time $t$. Then the fraction of cells hit by $n$ particles and visible in mitosis at time $t$ is $P(N=n) q^{n}(t)$. Accordingly, the p.m.f. of the random variable $N$ (representing number of charged particles traversing the cell nucleus) at time $t$ can be expressed in the form

$$
P_{t}(N=n)=\frac{P(N=n) q^{n}(t)}{\sum_{i=0}^{\infty} P(N=i) q^{i}(t)} .
$$

Following the assumption about the Poisson distribution of hits $N \sim \operatorname{Poisson}(\lambda)$, equation (8) can be rewritten as:

$$
P_{t}(N=n)=\frac{\frac{\lambda^{n} e^{-\lambda}}{n !} q^{n}(t)}{\sum_{i=0}^{\infty} \frac{\lambda^{i} e^{-\lambda}}{i !} q^{i}(t)}=\frac{\lambda q(t)^{n} e^{-\lambda q(t)}}{n !} .
$$

The corresponding probability generating function takes on the form

$$
F_{N}(s, t)=\sum_{n=0}^{\infty} \frac{\lambda q(t)^{n} e^{-\lambda q(t)}}{n !} s^{n}=e^{-\lambda q(t)(1-s)},
$$

so that

$$
E(N, t)=\lambda q(t)=\operatorname{Var}(N, t)
$$

By definition, the distribution of aberration is described by the compound Poisson process. Hence, its p.g.f. (1) assumes the form

$$
F_{S_{N}}(s, t)=e^{-\lambda q(t)\left(1-F_{X_{i}}(s)\right)}
$$

from which the first two moments can be evaluated, cf. equations (5) and (6):

$$
\begin{aligned}
E\left(S_{N}, t\right) & =\mu \lambda q(t), \\
\operatorname{Var}\left(S_{N}, t\right) & =\lambda q(t)\left(\sigma^{2}+\mu^{2}\right) .
\end{aligned}
$$

The above procedure, which undertakes $q(t)$ as a weighting factor in evaluation of the number of induced aberrations in hit cells was first proposed by Kaufman et al. [36] and Edwards et al. [13] to explain a low yield of aberrations appearing in mitoses at $48 \mathrm{~h}$ after exposure to alphaparticles. Note that the overall distribution of aberrations follows in this model a non-homogeneous compound Poisson distribution [17] with a rate $\Lambda=\lambda q(t)$.

\subsection{MI\&FCI Model (mitotic index and 1st cell cycle index model)}

More elaborate version of the above model takes into account analysis of subpopulations of hit and non-hit cells. Let $q(t)$ be the probability that a cell hit by one particle is able to reach mitosis at time $t$ and $z(t)$ stands for the probability that a non hit cell is in first mitosis at time point $t$. Then the fraction of cells hit by $n \geq 1$ particle and visible in mitosis at time $t$ is $P(N=n) q^{n}(t)$ and fraction of non hit cells visible at point $t$ is $P(N=0) z(t)$. Now, the p.m.f. of $N$ at time $t$ is:

$$
P_{t}(N=n)=\left\{\begin{array}{c}
\frac{P(N=0) z(t)}{P(N=0) z(t)+\sum_{i=1}^{\infty} P(N=i) q^{i}(t)}, n=0 \\
\frac{P(N=n) q^{n}(t)}{P(N=0) z(t)+\sum_{i=1}^{\infty} P(N=i) q^{i}(t)}, n>0
\end{array}\right\}
$$


and with the assumption $N \sim \operatorname{Poisson}(\lambda)$, can be rewritten to

$$
P_{t}(N=n)=\left\{\begin{array}{cc}
\frac{z(t)}{z(t)+e^{\lambda q(t)}}, & n=0 \\
\frac{(\lambda q(t))^{n}}{\left(z(t)+e^{\lambda q(t)}\right) n !}, & n>0 .
\end{array}\right.
$$

A corresponding p.g.f. for $N$-variable takes the form

$$
F_{N}(s, t)=\sum_{n=0}^{\infty} P_{t}(N=n) s^{n}=\frac{z(t)-1+e^{\lambda q(t) s}}{z(t)-1+e^{\lambda q(t)}}
$$

with the mean and variance given by:

$$
\begin{aligned}
E(N, t) & =\frac{\lambda q(t) e^{\lambda q(t)}}{z(t)-1+e^{\lambda q(t)}}, \\
\operatorname{Var}(N, t) & =E(N, t)(\lambda q(t)+1-E(N, t)) .
\end{aligned}
$$

Direct use of equation (1) implies the p.g.f. for the random sum $S_{N}$ at time $t$ :

$$
F_{S_{N}}(s, t)=\frac{z(t)-1+e^{\lambda q(t) F_{X_{i}}(s)}}{z(t)-1+e^{\lambda q(t)}} .
$$

Accordingly, mean and variance of $S_{N}$ (cf. Eqs. (5) and (6)) have simple expressions

$$
\begin{aligned}
E\left(S_{N}, t\right) & =E\left(X_{i}\right) E(N, t)=\mu \frac{\lambda q(t) e^{\lambda q(t)}}{z(t)-1+e^{\lambda q(t)}}, \\
\operatorname{Var}\left(S_{N}, t\right) & =E(N, t) \sigma^{2}+\mu^{2} \operatorname{Var}(N, t) .
\end{aligned}
$$

Note that in both $M I$ and $M I \& F C I$ models the timedependence in distribution of aberrations enters via kinetic parameters describing progression of cells through mitosis $q(t), z(t)$. In turn, parameters $\mu$ and $\sigma^{2}$ are constant in time mean and variance of the distribution of aberrations produced by a single particle traversal. Apparent departures of this distribution from a simple Poisson frequency, expected for induction of radiobiological effects by photons and low LET radiation, can be observed by studying dispersion parameter $r=$ $\operatorname{Var}\left(S_{N}, t\right) / E\left(S_{N}, t\right)$ which significantly deviates from 1 for non-Poisson statistics $[12,19]$.

Cells kinetics, i.e. their progression (or delay) in mitosis is well reflected experimentally by analysis of a mitotic index, $M I(t)$, which defines a time-dependent reaction rate for expanding population of dividing cells [28,29,37]. In experiments where the amount of chromosomal damage increases significantly with sampling time, a correction factor for dilution of late dividing, heavily damaged cells by the preceding division of undamaged or less damaged cells has to be applied [24,28]. Moreover, in order to constrain the analysis to the first post-irradiation cycle only, the total division rate $M I(t)$ has to be weighted with the fraction of cells at first mitosis. Both indices can be derived by applying a standard procedure of staining metaphase spreads by the FPG method, as described elsewhere $[8,10]$.

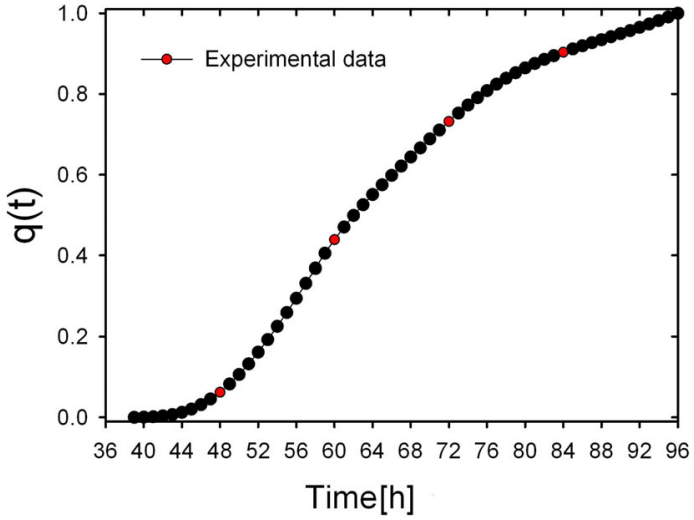

Fig. 6. Parameter $q(t)$ interpolated to fit experimental data collected at four different time points (red symbols).

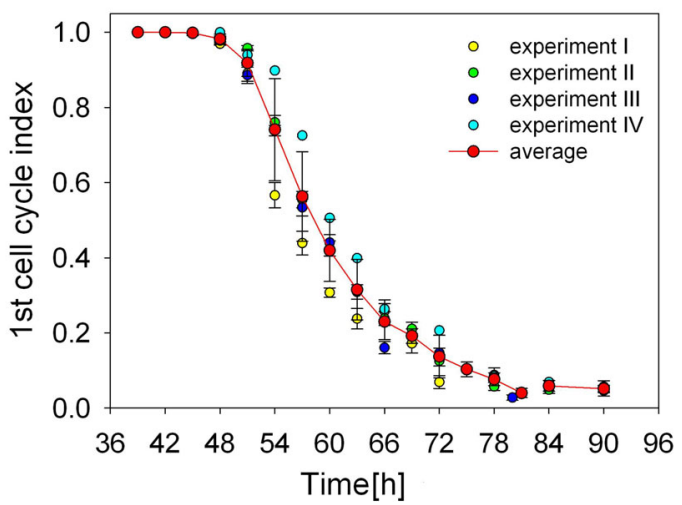

Fig. 7. Index of 1st cycle cells obtained from 4 independent experiments. Error bars represent intra-donor variability.

As the parameter $q(t)$, used in Section 5, we propose to hold the corrected mitotic index, as introduced by Scholz et al. [24,28,29]. Following this analysis, fraction of cells in first mitosis can be well approximated by the ratio of non-hit cells $z(t)$. The resulting expression for $q(t)$ is:

$$
q(t)=\int_{t_{0}}^{t} \frac{M I(t) z(t) \prod_{i=t_{0}}^{t}(1+M I(i))}{\int M I(t) z(t) \prod_{i=t_{0}}^{t}(1+M I(i)) d t} d t
$$

and has been displayed in Figure 6. The values $q(t)$ calculated from experimental data for 48,60,72 and $84 \mathrm{~h}$ after irradiation with $\mathrm{Fe}$ ions are illustrated as red symbols. Data for other time points have been interpolated by the Gaussian method with a cubic polynomial. In addition, Figure 7 displays parameter $z(t)$ derived from analysis of 4 control (non-irradiated) sets obtained from blood samples of the same donor.

\section{Comparison of models with experimental data}

In order to select the model of preference in description of metaphase transition through the cell cycle and analysis 


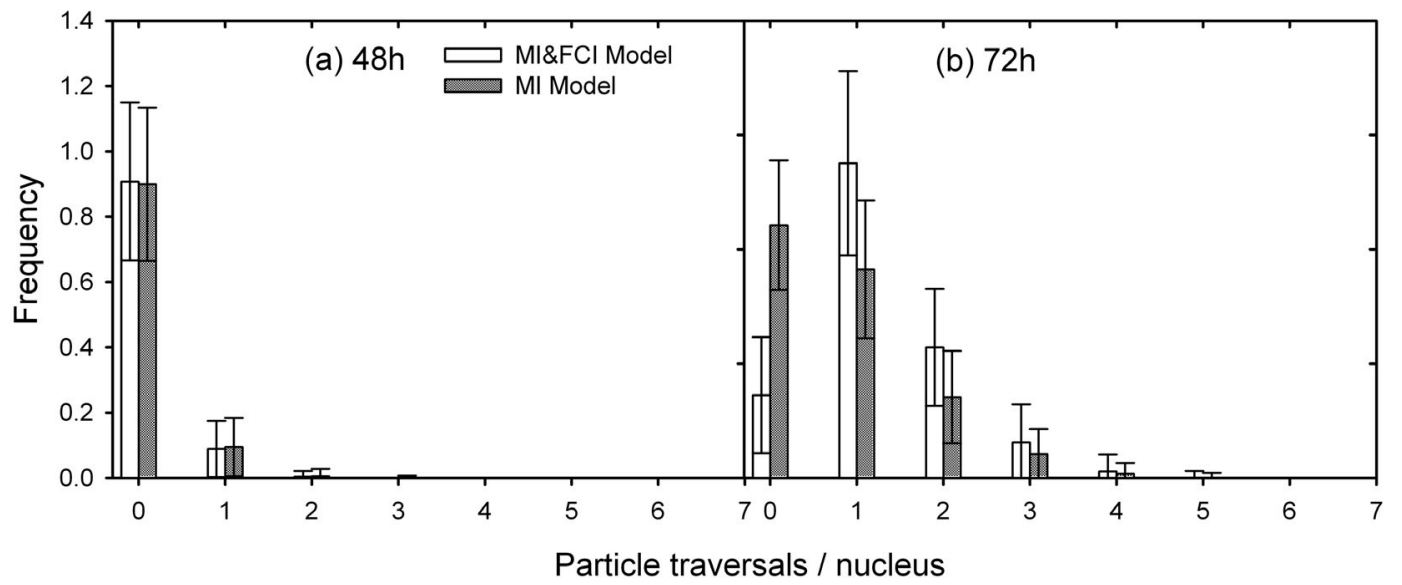

Fig. 8. Results of Monte Carlo simulations for 2.2 Gy $177 \mathrm{MeV} / \mathrm{u}$ Fe-ions (LET $=335 \mathrm{keV} / \mu \mathrm{m}$ ). Histograms represent distributions of the number of particle traversals in population of surviving cells at $48 \mathrm{~h}$ and $72 \mathrm{~h}$ after irradiation.

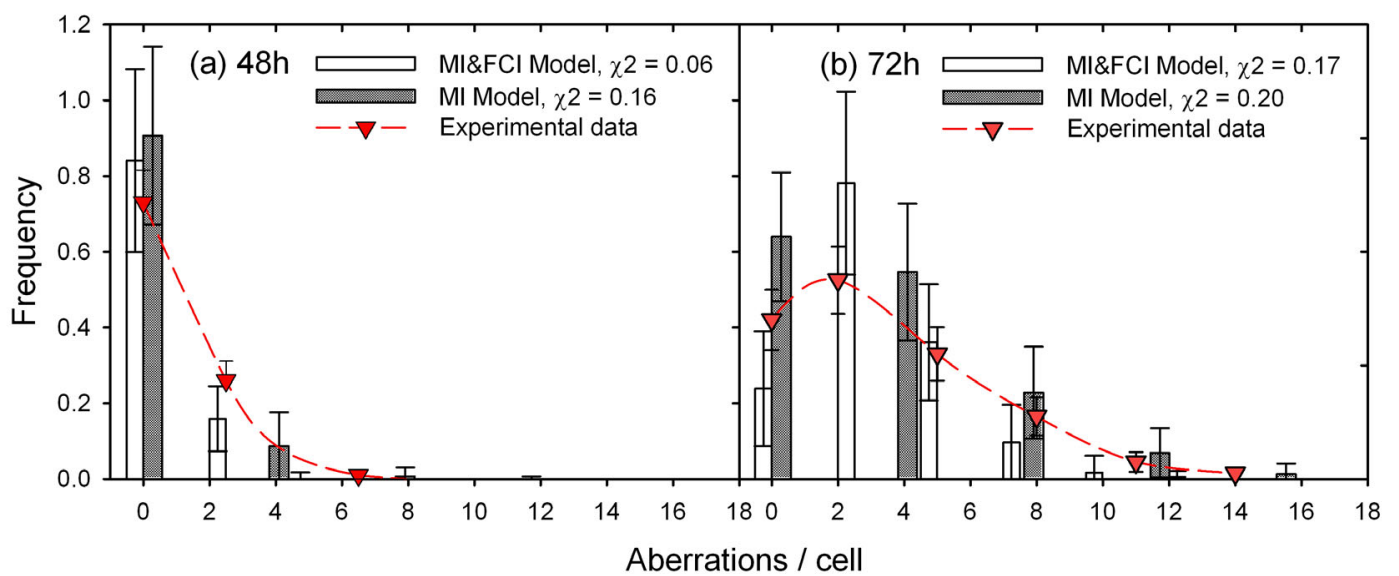

Fig. 9. Distribution of aberrations among cells as derived from Monte Carlo simulations for $2.2 \mathrm{~Gy} 177 \mathrm{MeV} / \mathrm{u}$ Fe-ions $(\mathrm{LET}=335 \mathrm{keV} / \mu \mathrm{m})$ and compared with experimental data.

of pattern of distribution of aberrations at different time points after irradiation, we have performed Monte Carlo (MC) simulations following the line of reasoning presented in Sections 5.1 and 5.2. The input parameters for the code are indices $q(t), z(t)$ and the value of $\lambda$ evaluated from the estimated fluence of ions (see Sect. 4.1). Unknown parameter $\mu$ is further derived in procedure of fitting simulated frequency of aberrations to experimental data obtained for 3-4 different time points. For each time point simulated frequency histograms involve repeated analysis of $10^{3}$ cells with subpopulations of aberrant and non-hit cells sampled from the compound Poisson distribution and deviations from the experimental values are checked by use of the $\chi^{2}$ test. The outcomes of the MC simulations for both models are presented in Figures 8 and 9 .

Clearly, the results derived from $M I \& F C I$ and $M I$ models differ for decreasing fraction of non-hit cells $z(t)$ completing first mitosis. For $z(t) \approx 1$ both approaches predict similar mean value of aberrations $E\left(S_{N}, t\right)$ detectable in cycling cells at a given time point $t$. Also, the parameter $\mu$, essential in estimation of $E\left(S_{N}, t\right)$ differs for fits to experimental data performed with either one of the procedures yielding $\mu=2.5 \pm 0.1$ and $\mu=3.8 \pm 0.2$ for $M I \& F C I$ and $M I$ model, respectively. Accordingly, Figure 9 displays differences in predicted distributions of aberrations derived from simulated data.

Additionally, our analysis provides means to construct expected distribution of aberrations produced by one particle traversal through the cell nucleus, $P\left(X_{i}=x\right)$. Following procedure described in Section 3 , for each time point $t$, the statistics of the random variable $S_{N}$ can be deduced from recurrent use of equations (3) and (4). Equivalently, those equations can be used to derive $P\left(X_{i}=x\right)$ provided known statistics of $P\left(S_{N}=k\right)$ and $P_{t}(N=n)$. Data obtained in this way have been illustrated in Figures 10 and 11.

Although frequency histograms predicted by both models equally well reflect experimental distributions of aberrations among 100 cells, the MI\&FCI model seems to be more representative in fitting the value of $\mu$ parameter (the number of aberrations per one particle hit) which stays closer to the experimental estimate [9]. Further 


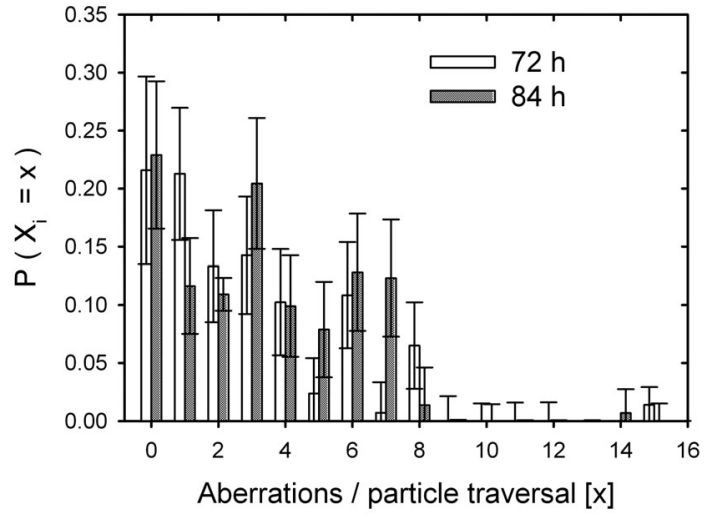

Fig. 10. Distribution of aberrations produced by one particle hit $P\left(X_{i}=x\right)$ derived from MC distributions of aberrations in 100 cells $P\left(S_{N}=k\right)$ fitted to experimental data at $72 \mathrm{~h}$ and $84 \mathrm{~h}$. Cells were exposed to 2.2 Gy $177 \mathrm{MeV} / \mathrm{u}$ Fe-ions $(\mathrm{LET}=335 \mathrm{keV} / \mu \mathrm{m})$. Error bars have been estimated by the method of propagation of uncertainty.

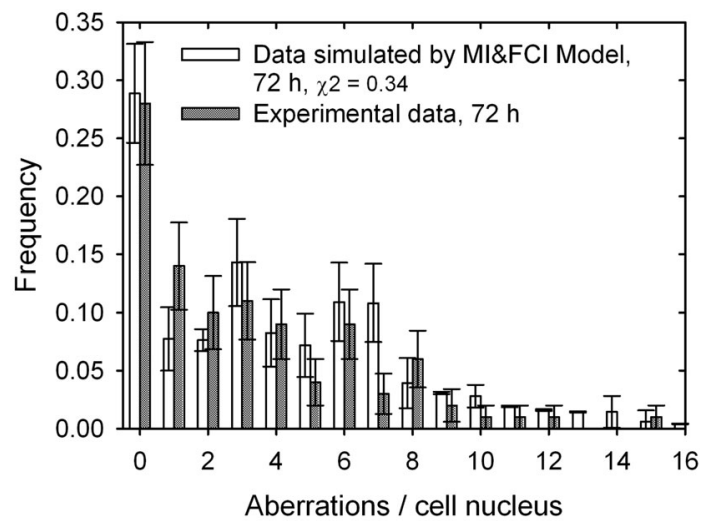

Fig. 11. Experimental distribution of aberrations in irradiated cells at $72 \mathrm{~h}$ after exposure compared with analogous frequency deduced from the Monte Carlo MI\&FCI model. Error bars as in Figure 9.

analysis of aberration yield and distribution after exposure to other ions should be applied to confirm this point.

In conclusion, we have presented a detailed stochastic model which accurately describes cytogenetic effects of densely ionizing radiation by analysis of pattern of distribution of aberrations among cells. The analysis contributes substantially to manner of interpretation of cytogenetic data with respect to the particular scenario of radiation exposure. For example, a Poisson distribution of damage may be representative for uniform radiation exposure, whereas more complex, compound Poisson distributions with a high overdispersion index are indicative for irradiation with charged particles. When additionally weighted with the reaction rate describing transmission of the first cycle cells through mitosis, the model correctly describes flow of aberrant cells in irradiated population and allows to predict aberration yield at various postexposure times.
This work is supported by the International $\mathrm{PhD}$ Projects Programme of the Foundation for Polish Science within the European Regional Development Fund of the European Union, agreement No. MPD/2009/6. E.G.-N. acknowledges additional support from the European Science Foundation grant Exploring Physics of Small Devices.

\section{References}

1. J.D. Tucker, Mutat. Res. 659, 211 (2008)

2. A. Wojcik, D. Lloyd, H. Romm, L. Roy, Radiat. Prot. Dosim. 138, 397 (2010)

3. M.M.P.L. Pinto, N.F.G. Santos, A. Amaral, Radiat. Environ. Biophys. 49, 567 (2010)

4. M. Durante, F. Cucinotta, Nat. Rev. Cancer 8, 465 (2008)

5. F.A. Cucinotta, W. Schimmerling, J.W. Wilson, L.E. Peterson, G.D. Badhwar, P.B. Saganti, J.F. Dicello, Radiat. Res. 156, 682 (2001)

6. J.L. Huff, F.A. Cucinotta, in Human Health and Performance Risks of Space Exploration Missions, Evidence reviewed by the NASA Human Research Program, edited by J. McPhee, J.B. Charles (NASA, Lyndon B. Johnson Space Center, Houston, USA, 2009), No. NASA SP-2009-3405, pp. 213-235

7. M. Durante, Y. Furusawa, E. Gotoh, Int. J. Radiat. Biol. 74, 457 (1998)

8. S. Ritter, E. Nasonova, M. Scholz, W. Kraft-Weyrather, G. Kraft, Int. J. Radiat. Biol. 69, 155 (1996)

9. R. Lee, E. Nasonova, C. Hartel, M. Durante, S. Ritter, Radiat. Environ. Biophys. 50, 371 (2011)

10. S. Ritter, E. Nasonova, E. Gudowska-Nowak, Int. J. Radiat. Biol. 78, 191 (2002)

11. J. Deperas-Standylo, R. Lee, E. Nasonova, S. Ritter, E. Gudowska-Nowak, Adv. Space Res. 50, 584 (2012)

12. S. Karlin, H. Taylor, A First Course in Stochastic Processes (Academic Press, London, 1975)

13. A. Edwards, R. Purrott, J. Prosser, D. Lloyd, Int. J. Radiat. Biol. 38, 83 (1980)

14. M. Krämer, G. Kraft, Radiat. Environ. Biophys. 33, 91 (1994)

15. H. Nikjoo, S. Uehara, D. Emfietzoglo, A. Brahme, New J. Phys. 10, 075006 (2008)

16. M. Scholz, G. Kraft, Adv. Space Res. 18, 5 (1996)

17. J. Neyman, P. Puri, Proc. Natl. Acad. Sci. USA 73, 3360 (1976)

18. M.J. Dobbie, A.H. Welsh, Stat. Model. 1, 65 (2001)

19. E. Gudowska-Nowak, E. Nasonova, S. Ritter, M. Scholz, Radiother. Oncol. 73, S123 (2004)

20. M. Scholz, Adv. Polym. Sci. 162, 95 (2003)

21. B. Jakob, J. Splinter, M. Durante, G. Taucher-Scholz, Proc. Natl. Acad. Sci. USA 106, 3172 (2009)

22. R. Anderson, S. Marsden, E. Wright, M. Kadhim, D. Goodhead, C. Griffin, Int. J. Radiat. Biol. 76, 31 (2000)

23. G. Hoffmann, A.M. Sayer, L.G. Littlefield, Int. J. Radiat. Biol. 78, 765 (2002)

24. S. Ritter, E. Nasonova, Y. Furusawa, K. Ando, J. Radiat. Res. (Tokyo) Suppl. 43, 175 (2002)

25. R. Lee, E. Nasonova, S. Ritter, Adv. Space Res. 35, 268 (2005)

26. E. Gudowska-Nowak, R. Lee, E. Nasonova, S. Ritter, M. Scholz, Adv. Space Res. 39, 1070 (2007) 
27. J. Deperas-Standylo, R. Lee, A. Ayriyan, E. Nasonova, S. Ritter, E. Gudowska-Nowak, Eur. Phys. J. D 60, 93 (2010)

28. M. Scholz, S. Ritter, G. Kraft, Int. J. Radiat. Biol. 74, 325 (1998)

29. E. Gudowska-Nowak, A. Kleczkowski, E. Nasonova, M. Scholz, S. Ritter, Int. J. Radiat. Biol. 81, 23 (2005)

30. S. Tenhumberg, E. Gudowska-Nowak, E. Nasonova, S. Ritter, Int. J. Radiat. Biol. 83, 501 (2007)

31. E. Nasonova, S. Ritter, Cytogenet. Genome Res. 104, 216 (2004)

32. E. Nasonova, S. Ritter, E. Gudowska-Nowak, G. Kraft, Phys. Med. 17, 198 (2001)

33. E. Gudowska-Nowak, A. Kleczkowski, G. Kraft, E. Nasonova, S. Ritter, M. Scholz, Phys. Med. 17, 161 (2001)
34. A. Ochab-Marcinek, E. Gudowska-Nowak, E. Nasonova, S. Ritter, Radiat. Environ. Biophys. 48, 361 (2009)

35. A. Edwards, Int. J. Radiat. Biol. 78, 551 (2002)

36. G. Kaufman, A. Miller, J. Savage, D. Papworth, J. Theor. Biol. 44, 91 (1974)

37. K. George, H. Wu, V. Willingham, Y. Furusawa, T. Kawata, F. Cucinotta, Int. J. Radiat. Biol. 77, 175 (2001)

Open Access This is an open access article distributed under the terms of the Creative Commons Attribution License (http://creativecommons.org/licenses/by/4.0), which permits unrestricted use, distribution, and reproduction in any medium, provided the original work is properly cited. 